\title{
INFLUENCE OF THE APPLICATION OF LIGNITE ON THE DISTRIBUTION OF ORGANIC CARBON IN SOIL
}

\author{
David ŠIRŮČEK, Michal KALINA, Martina KLUČÁKOVÁ \\ Brno University of Technology, Faculty of Chemistry, Brno, Czech Republic, EU
}

https://doi.org/10.37904/nanocon.2020.3765

\begin{abstract}
This contribution is focused on utilization of a sequential chemical fractionation method for determination of organic matter content and its distribution in soil. Subsequently, this fractionation procedure was used to characterize the possible use of lignite as a soil conditioner in agriculture. For these purposes the optimized chemical fractionation procedure was used to describe the effect of lignite application on content and distribution of organic matter in a model soil. For these purposes, there were three source matrices of organic matter (lignite, soil and annual soil extraction after lignite application) fractionated by sequential chemical analysis. In parallel, these samples were also fractionated by classic alkaline extraction to obtain the so-called extractable fraction of organic matter (NOM). Individual fractions from sequential chemical fractionation as well as NOM samples were characterized by methods of elemental analysis (determination of organic elements content), thermogravimetry (contents of ash, organic matter and moisture) and FTIR spectrometry (structural analysis). The results showed that the method of sequential chemical fractionation gives higher yields of organic matter compared to classical alkaline extraction. Moreover, the obtained fractions divided according to solubility and strength of binding to soil inorganics can be better characterized by physical-chemical methods, which provides more detailed information about soil organic matter. The results of the work also indicated that the effect of lignite on soil organic matter must be considered in a larger time scale to be able to get more complex description of such a complex system.
\end{abstract}

Keywords: Soil, lignite, organic matter, chemical fractionation, elemental analysis, thermogravimetric analysis, FTIR spectrometry

\section{INTRODUCTION}

Soils are composed of mineral and organic particles of various sizes, that are organized in a soil matrix. This organization means that about $50 \%$ of the total volume is occupied by these particles and the remaining $50 \%$ of the volume is a porous space that contains water and air and serves as a system of channels for the transport of these substances. Soil organic matter (SOM) is one of the soil components consisting of three basic types - living organisms (worms, insects, microorganisms), dead microorganisms and finally matter formed from organic residues of plants, animals and humus. The decomposition of the first two types creates nutrients for plants (nitrogen, potassium, phosphorus) [1-3]. The main reactive part of SOM are humic substances omnipresent natural substances arising from the chemical and biological degradation of plants and animal remains. They are crucial in ecosystems because they regulate global carbon and nitrogen cycles, the growth of plants and microorganisms, the transport of anthropogenic compounds and heavy metals, and the stabilization of soil structure. To date, however, no technologies have been developed to control the activity of humic substances due to their high molecular complexity. There is a broad variety of analytical techniques for their characterization, however, none of these techniques alone is sufficient to elucidate the structural complexity of particles of humic molecules [4,5].

In general, humic substances are supramolecular associations of heterogeneous, relatively small molecules that are held together by weak non-covalent interactions, such as hydrogen bonds and hydrophobic forces. 
The complexity of the humic molecule can be reduced by the gradual destruction of intramolecular interactions that stabilize these complex suprastructures (composed of nanoparticles of organic matter) and thus individual fractions of colloidal size of humic substances (organic matter) can be isolated. These individual fractions of organic matter obtained from sequential fractionation are called humeomics. It is a gradual separation of molecules from humic suprastructures by controlled cleavage of intermolecular bonds. In terms of particle size, these particles obtained by sequential fractionation are colloidal in size which significantly improve the resolution of various analytical methods often used for characterization $[6,7]$.

In recent years, increasing demands have been placed on crop yields which also brings with it increased demands on soil fertility. For these reasons, various substances are often used to increase fertility, which are added artificially. They are most often fertilizers. Recently, however, research has also focused on the application of alternative additives to soils in order not only to increase fertility but also to improve the physicochemical properties of the soil and to increase the content and stabilization of soil organic matter. These additives are often included in a group called - soil supportive substances (SSS). Substances that are widely used for application include, for example, lignite fly ash, biochar and a mixture of humic acids. It is also very important to apply a suitable form with a given particle size, internal structure and morphology (porous structure) $[8,9]$.

\section{EXPERIMENTAL}

\subsection{Materials and Methods}

Three source matrices of organic matter were used.

- $\quad$ Soil (arid sandy soil obtained from the locality Hodonin-Panov, GPS: $48.877184^{\circ} \mathrm{N}, 17.132040^{\circ} \mathrm{E}$ )

- $\quad$ Lignite (South Moravian lignite, mine Mir near Mikulcice, GPS: $48.820864^{\circ} \mathrm{N}, 17.045283^{\circ} \mathrm{E}$ )

- Soil sample after one year from lignite application (the same locality as original soil)

Each of these organic matrix samples served as a starting material for studying the effect of lignite application on the distribution of organic matter in the soil. For these purposes, the method of sequential chemical fractionation was used in the work. The initial samples of lignite and soil were used mainly as comparative samples, on the basis of which the differences in the distribution of organic matter in the soil before and after the application of lignite as a model soil support substance will be assessed. Three replicates of sequential chemical fractionation were performed for each of these three samples.

\subsection{Sequential Chemical Fractionation}

The procedure of sequential chemical fractionation was adopted from the publications of Nebbioso and Drossos [6,7]. The details of the optimization of this chemical extraction procedure of organic matter for the samples of soil and lignite are summarized in chapters 2.2.1 - 2.3.

\subsubsection{Water soluble fraction 1 (AQU1)}

First step was to dry used organic material in an oven at $45^{\circ} \mathrm{C}$ to evaporate all the water. Then $50 \mathrm{~g}$ of dried material was transferred to Erlenmeyer flask and $150 \mathrm{ml}$ of $0,1 \mathrm{M} \mathrm{HCl}$ was added. Flasks were covered with a double layer of parafilm and left on a shaker overnight. The next day samples were filtered under reduced pressure. Filtrate was frozen and all the water was evaporated in a freeze drier.

\subsubsection{Organic soluble fraction 1 (ORG1)}

Dried solid material after AQU1 was mixed with $100 \mathrm{ml} \mathrm{DCM}$ and $50 \mathrm{ml} \mathrm{MeOH}$ in Erlenmeyer flask and covered with a double layer of aluminium foil and left on a shaker overnight. The next day samples were filtered under 
reduced pressure. All the solvent was evaporated on a rotary evaporator at $50{ }^{\circ} \mathrm{C}$. The residue after evaporation was transferred to vials left to evaporate the solvent.

\subsubsection{Water and organic soluble fractions 2 (AQU2 and ORG2)}

Dried solid material after ORG1 separation was transferred to high-pressure sealable teflon vials and $50 \mathrm{ml}$ of a $12 \%$ solution of boron trifluoride in methanol was added. Vials were tightly sealed, clamped in a metal rack and placed in an oven at $85{ }^{\circ} \mathrm{C}$ for 8 hours. Then vials were removed from the oven, let to cool down and additional $50 \mathrm{ml}$ of the solution was added. Vials were sealed and clamped again and placed in the oven for another 8 hours under same conditions. Then after cooling down the solution was filtered under normal pressure and the solid material was dried. The filtrates were L/L extracted (liquid to liquid). The obtained AQU2 and ORG2 fractions were purified and dried in the same way as was described for AQU1 and ORG1.

\subsubsection{Water and organic soluble fractions 3 (AQU3 and ORG3)}

Dried solid material after the previous step was covered with $50 \mathrm{ml}$ of $1 \mathrm{M}$ solution of $\mathrm{KOH}$ in $\mathrm{MeOH}$ (for the lignite sample a $100 \mathrm{ml}$ of the solution was used). A magnetic stirrer was added, the sample flask was attached to a direct condenser and heater was set to $75^{\circ} \mathrm{C}$. The mixture was refluxed under nitrogen atmosphere for 2 hours. Then all the content in the flask was filtrated under reduced pressure and the solid material was dried. The filtrates were extracted using L/L extraction. The obtained AQU3 and ORG3 fractions were purified and dried in the same way as was described for AQU1 and ORG1.

\subsubsection{Water soluble fraction 4 (AQU4)}

Dried solid material after the previous step was covered with $50 \mathrm{ml}$ of a $47 \%$ solution of $\mathrm{HI}$ in water (for the lignite sample a $100 \mathrm{ml}$ of the solution was used). A magnetic stirrer was added, the sample flask was attached to a direct condenser and heater was set to $75^{\circ} \mathrm{C}$. The mixture was refluxed under nitrogen atmosphere for 2 days. Then all the content in the flask was filtrated under reduced pressure and the solid material was dried. The filtrates were neutralized with $\mathrm{NaHCO}_{3}$ to $\mathrm{pH} 7$ and the iodine contained was reduced with $\mathrm{Na}_{2} \mathrm{~S}_{2} \mathrm{O}_{3}$. Then the filtrates were dialyzed against distilled water and freeze-dried.

\subsubsection{Residual extractable organic matter fraction (ResOM)}

To isolate the residual organic matter, $5 \mathrm{~g}$ of dried solid material after AQU4 fractionation and $45 \mathrm{ml}$ of extractant $\left(0,5 \mathrm{M} \mathrm{NaOH}\right.$ and $\left.0,1 \mathrm{M} \mathrm{Na}_{4} \mathrm{P}_{2} \mathrm{O}_{7}\right)$ were mixed. Samples were left on a shaker overnight. The samples were filtered and the filtrates were dialyzed against distilled water and freeze-dried.

\subsection{Natural organic matter fraction isolation (NOM)}

In parallel, a conventional alkaline extraction was performed. Thus $5 \mathrm{~g}$ of the prime material was covered with $45 \mathrm{ml}$ of extractant $\left(0,5 \mathrm{M} \mathrm{NaOH}\right.$ and $\left.0,1 \mathrm{M} \mathrm{Na}_{4} \mathrm{P}_{2} \mathrm{O}_{7}\right)$. After overnight shaking, the samples were filtered under reducer pressure and the filtrates were dialyzed against distilled water and freeze-dried.

\section{RESULTS AND DISCUSSION}

\subsection{Optimization of sequential chemical fractionation procedure}

The first part of experimental work was focused on the optimization of sequential chemical fractionation for analysis of soil and lignite samples to be able to provide more narrow distribution of particles. A comparison shown in Figure 1 shows that only about a half on the organic matter can be extracted using NOM extraction (conventional alkaline extraction). But as shown in Figure 1a, only a very small amount of organic matter (about $10 \%$ ) was possible to obtain using sequential chemical fractionation. Comparing each fraction shown in Figure 1b, we may notice that most of the organic matter is contained within AQU1 and ORG1 fractions. 
That is because these two fractions are only very weakly bounded to the lignite matrix. These results are indicating that significant part of lignite organic matter is strongly bound to residual non-extractable matter. This is a positive finding concerning the presumed long-term effect of lignite on the organic matter of the soil.
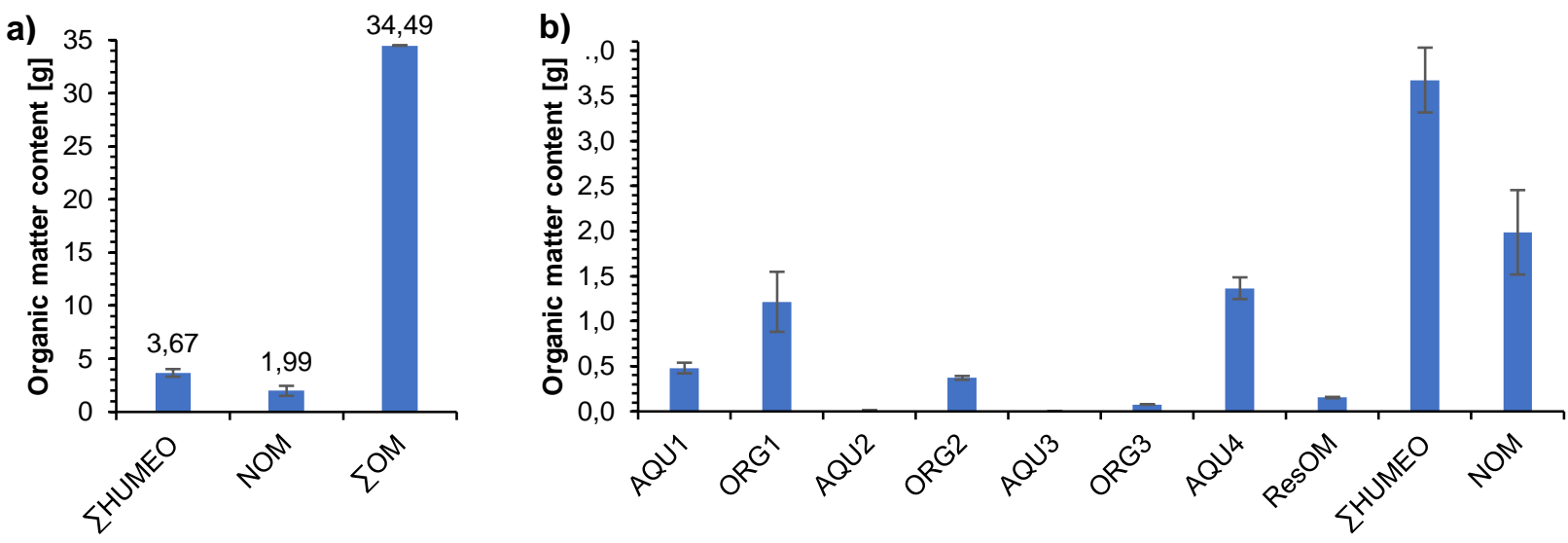

Figure 1 a) Comparison of total organic matter from lignite using alkaline extraction and sequential chemical fractionation where " $\Sigma$ HUMEO" means the total sum of the particle fractions obtained by sequentional fractionation and " $\Sigma O M$ " shows the total contain of organic matter contained in the soil matrix. This applies to all the graphs. b) Distribution of organic matter in the fractions of a lignite sample.

\subsection{Effect of lignite application on soil}

The main task of this part of the work was to use the methodology of sequential chemical fractionation to assess the effect of the addition of lignite to the soil on the composition and distribution of organic matter. In general, the organic matter content in lignite is much higher than in the soil matrix, however, across the fractions of humeomics, the difference in organic matter content between the matrices varies.
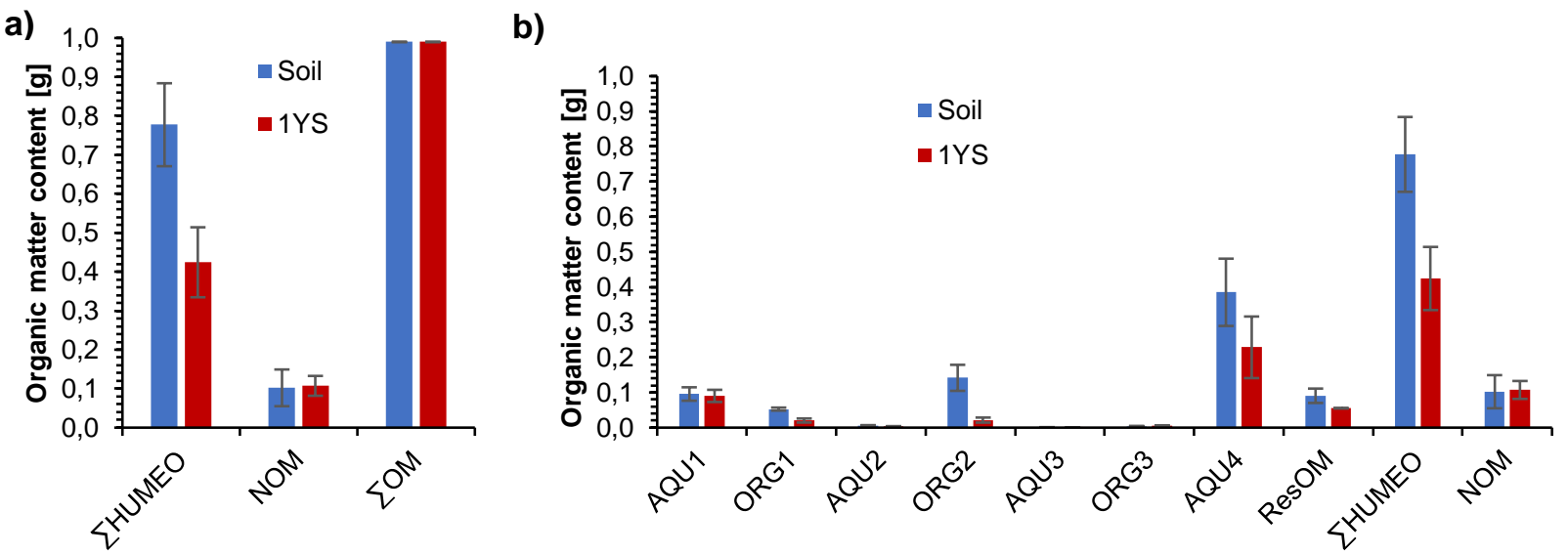

Figure 2 a) Comparison of both (conventional alkaline extraction and sequential chemical fractionation) methods with the total amount of organic matter in soil and 1YS (one year sample). b) Comparison of organic matter content in all fractions obtained from the soil and 1YS (one year sample) matrices

After closer examination of the individual fractions, it is visible that the contents of organic matter in the original soil matrix are higher than in the annual soil sampling. However, this contradicts the theoretical assumption that the proportion of organic matter should increase over time after application of the adjuvant. However, if we compare obtained content of organic matter by classical alkaline extraction NOM, the original sample and annual sampling are practically comparable. The decrease observed above in total yield of organic matter obtained on the soil sample after one year compared to the yield from the fractionation of the original soil 
sample before lignite application is probably due to the heterogeneity of the soil sample and the relatively high content of sand fractions.

However, if we compare extractable NOM and a sum of organic matter obtained from sequential chemical fractionation, it is clearly visible that with sequential chemical fractionation we can extract several times higher amount of organic matter than the NOM method. However, the difference in the content of extracted organic matter by both methods is more noticeable in soil matrices than in lignite matrices, which may be related to the total content of organic matter in the matrices.

\subsection{FTIR structure of fractions}

FTIR was used as a simple method for assessment of structural motifs in all the obtained fraction of relatively similar particles organic matter. The FTIR spectra of the ORG1 fractions in Figure 3a contain a peak in the region of 3,650-3,000 $\mathrm{cm}^{-1}$, which lies in the area where $\mathrm{N}-\mathrm{H}$ bonds, phenols and alcohols should occur. Furthermore, from $3,000-2,800 \mathrm{~cm}^{-1}$, a significant peak appears marking the saturated $\mathrm{C}-\mathrm{H}$ bonds of alkanes. In the area of 2,200-1,950 $\mathrm{cm}^{-1}$ we find overtones. This is followed by a pair of peaks in the range of 1,900 $1,550 \mathrm{~cm}^{-1}$, which indicates carbonyl bonds, and between $1,550-1,450 \mathrm{~cm}^{-1}$, are present aromatic compounds. A peak with a maximum at about $1,050 \mathrm{~cm}^{-1}$ refers to aliphatic $\mathrm{O}-\mathrm{H}$ bonds of alcohols, and the last peak with a maximum of about $800 \mathrm{~cm}^{-1}$ indicates the deformation transition in aromatics. For more details see $[6,7]$.
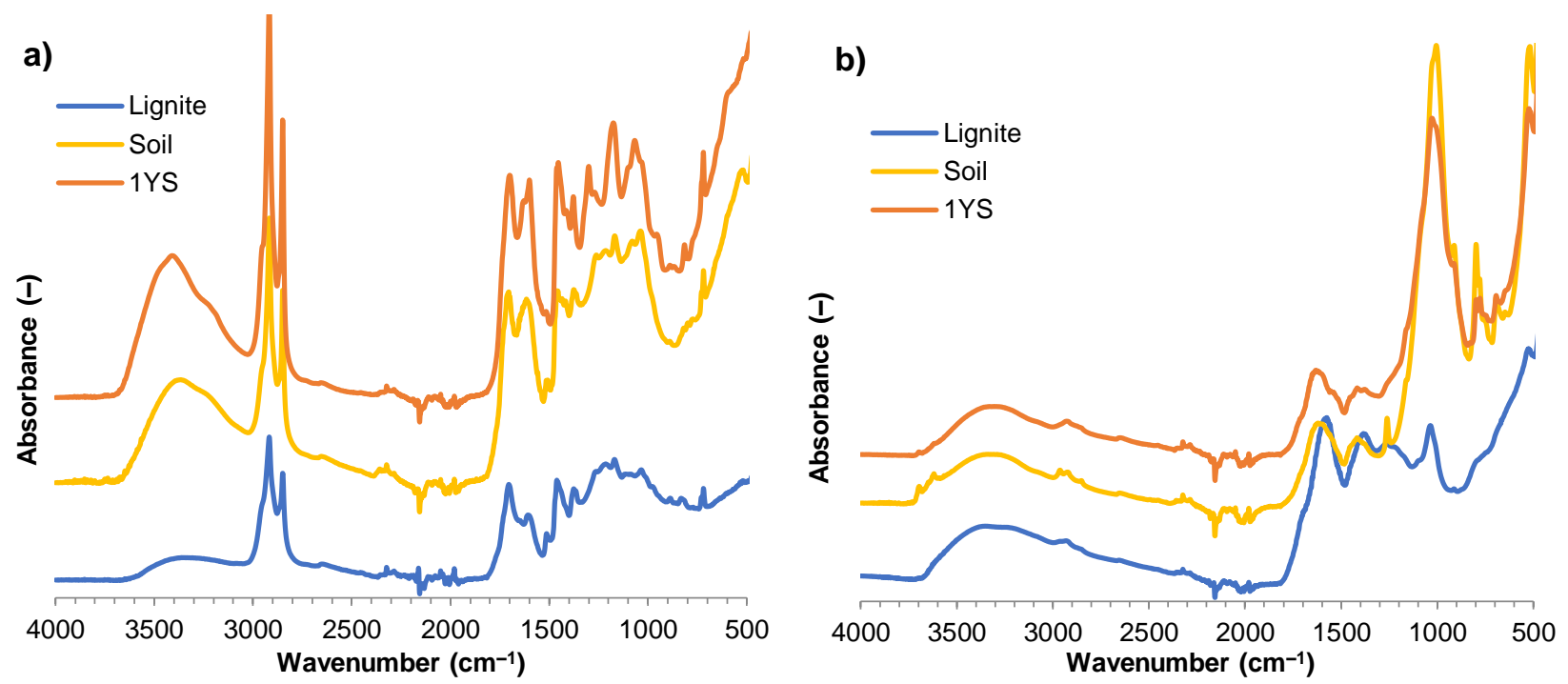

Figure 3 a) FTIR spectra of ORG1 fractions for all the source matrices. b) FTIR spectra of NOM fractions for all the source matrices

From the FTIR spectrum of the NOM fractions (Figure $3 \mathbf{b}$ ) is obvious that when we try to characterize the total isolated organics, heterogeneity and the number of different molecules are a huge problem. Sequential chemical fractionation makes it possible to obtain smaller fractions with relatively narrow distribution of molecules, which makes the subsequent characterization easier. This resulted in the possibility to use FTIR for identification of several different types of substances present in the fractions.

\section{CONCLUSION}

This contribution was focused on optimization of a sequential chemical fractionation for purposes of a study on the effect of lignite (and possibly also other agriculturally attractive SSS) application on SOM, its total content and distribution in the soil. The procedure provided set of organic matter fractions with different strength of the interaction to the remaining inorganic skeleton and also with different polarity of molecules. 
The obtained fractions of organic matter were characterized by elemental (mainly content of carbon and nitrogen essential for plant growth) and thermogravimetric analysis (ash, organic matter and moisture content). The results showed that a shift in the distribution carbon and nitrogen, which are essential for plant nutrition and proper soil function, was observed only in the first isolated fractions (AQU1 and ORG1), which are not firmly bound to the matrix structure (composed of nanoparticles of organic matter) and therefore are the most accessible to plants. In other fractions, no changes were observed, suggesting that the time horizon of one year is very short to monitor the changes induced by the application of lignite to the soil and is not sufficient to induce large changes deeper in the matrix structure. The FTIR spectrometry used subsequently was confirmed to be usable for monitoring of the structural changes in the individual fractions and to approximately define groups of substances that were obtained in individual fractions.

The results of the work confirmed that in order to properly assess the effect of soil support application on the content and distribution of organic matter in soil, it is necessary to study samples obtained over a longer period of time from soil supporting substance application. Also this paper assesses the effect of application of lignite on soil organic matter but of course the application form and therefore the particle size or internal structure (texture) is essential and it will be the subject of further follow-up research.

\section{ACKNOWLEDGEMENTS}

This work has been supported by project GJ20-28208Y from Czech Science Foundation and by project "Materials Research Centre at FCH BUT - Sustainability and Development" No. LO1211 of the Ministry of Education, Youth and Sports of the Czech Republic.

\section{REFERENCES}

[1] Agronomy Fact Sheet no. 41: Soil organic matter. Cornell University Publications, 2008. [cit. 2014-01-21].

[2] PAUL, E. A., CLARK, F. E. Soil Microbiology and Biochemistry. San Diego: Academic Press, 1996. p. 340.

[3] TAN, K.H. Humic Matter in Soil and the Environment. Principles and Controversies. New York: Marcel Dekker, Inc., 2003, p. 386.

[4] GUSTAFSON, A. F. Soils and soil management (McGraw-Hill publications in the agricultural sciences). London: McGraw-Hill Book Company, 1941.

[5] BOHLEN, P. J.; EDWARDS, C. A. Earthworm effects on N dynamics and soil respiration in microcosms receiving organic and inorganic nutrients. Soil Biology and 50 Biochemistry. 1995, vol. 27, pp. 341-348. [cit. 2017-02-26].

[6] NEBBIOSO, A., PICCOLO, A. Basis of a Humeomics Science: Chemical Fractionation and Molecular Characterization of Humic Biosuprastructures. Biomacromolecules. 2011, vol. 12, no. 4, pp. 1187-1199. [cit. 2019-03-31].

[7] DROSOS, M., NEBBIOSO, A., MAZZEI, P., VINCI, G., SPACCINI, R., PICCOLO, A. A molecular zoom into soil Humeome by a direct sequential chemical fractionation of soil. Science of The Total Environment. 2017, vol. 586, pp. 807-816.

[8] MASTO, R. ebhin, Md. a. ANSARI, J. GEORGE, V.a. SELVI a L.c. RAM. Co-application of biochar and lignite fly ash on soil nutrients and biological parameters at 77 different crop growth stages of Zea mays. Ecological Engineering. Elsevier B.V. 2013, vol. 58, pp. 314-322. [cit. 2019-04-05].

[9] RAM, L., SRIVASTAVA, N., JHA, S., SINHA, A., MASTO, R., SELVI, V. Management of Lignite Fly Ash for Improving Soil Fertility and Crop Productivity. Environmental Management. New York: Springer-Verlag, 2007, vol. 40, no. 3, pp. 438-452. [cit. 2019-04-07]. 